N. Lehn

\title{
Teilnahmebedingungen
}

Für diese Fortbildungseinheit erhalten Sie drei Fortbildungspunkte. Hierfür

- müssen 9 der 11 Quiz-Fragen richtig beantwortet sein (bestanden hat auch, wer nicht weniger Fragen richtig beantwortet hat als der Durchschnitt aller Teilnehmer).

- müssen Seite 1 und Seite 2 des Evaluationsbogens vollständig ausgefüllt sein. Unvollständig ausgefüllte Bögen können nicht berücksichtigt werden!

- muss eine CME-Wertmarke im Feld D (Evaluationsbogen) aufgeklebt oder Ihre DMW-Abonnement-Nummer im Feld D eingetragen bzw. über das Internet eingegeben sein.

- Einsendeschluss ist der 18.5.2005

(Datum des Poststempels bzw. Eingangsdatum der E-Mail). Die Teilnahme-Bescheinigungen werden ab dem 25.5.2005 versandt. Teilnehmer auf dem Postweg erhalten ihre Bescheinigung per Post, Online-Teilnehmer per E-Mail. Von telefonischen Anfragen bitten wir abzusehen.

Die richtige Auflösung des Quiz wird in der DIMW/ Nr. 21/2005 (erscheint am 27.5.2005) veröffentlicht.

\section{Methicillin-resistenter Staphylococcus aureus (MRSA)}

\section{Eine und nur eine Antwort ist jeweils richtig!}

Welche Aussage ist richtig?
Der Nachweis von MRSA

kann erfolgen durch...
A ... Kultur und anschließende Resistenztestung gegen Penizillin mittels Blättchendiffusion.

B ... Nachweis des mecA-Gens mittels Multi-Locus-Sequenz-Typisierung.

C ... Nachweis des alternativen Penicillin-Bindeproteins PBP2a aus Kultur von S. aureus.

D ... Nachweis des alternativen Penicillin-Bindeproteins PBP2a direkt aus klinischem Material.

E ... Bestimmung der minimalen Hemmkonzentration gegen Penicillin.

A nekrotisierende Fasziitis

B Parotitis

C atypische Pneumonie

D ambulant erworbene Meningitis

E Abszesse

A Rifampicin

B Fusidinsäure

C Makrolide

D Fosfomycin

E Linezolid

A Epithelien

B Granulozyten

C Fibroblasten

D Lymphozyten

E Erythrozyten
Welche Aussage ist richtig? Die wichtigste Hygienemaßnahme bei MRSA ist ...
... Einzelzimmer mit Schleuse und Unterdruck.

B ... Schutzkittel für Personal und Besucher.

C ... Händedesinfektion bei Verlassen des Zimmers.

D ... Mund-Nasenschutz für Personal.

E ... Wischdesinfektion des Fußbodens. 
Zur Behandlung des

Trägerstatus von MRSA ist/ sind geeignet:

Die Beendigung von

Hygienemaßnahmen möglich nach ... Die Kombination von Vancomycin mit Rifampicin oder Fusidinsäure wird empfohlen... Übertragungsweg von

Die am wenigsten wirksame Strategie zur Verhütung von durch MRSA ist...
A Therapie mit Vancomycin

B Therapie mit Vancomycin in Kombination mit externer Anwendung antimikrobieller Waschlösungen

C Mupirocin-Nasensalbe

D Mupirocin-Nasensalbe für Nase und Wunden

E Rifampicin (Dosierung wie bei Prophylaxe gegen Meningokokken)

A Vancomycin

B Linezolid

C Daptomycin

Duinopristin/Dalfopristin

E Vancomycin + Rifampicin oder Fusidinsäure

Welche Aussage ist richtig? A ... negativem Nachweis mittels Nasenabstrich. wegen MRSA-Nachweis ist

B ... Durchführung von Maßnahmen zur Sanierung des Trägerstatus wie Mupirocin-Nasensalbe und Anwendung von externen antimikrobiellen Waschlösungen.

C ... einer Therapie mit Vancomycin.

D ... dreimaligem negativem Nachweis aus Nase und ggf. anderen in Vorbefunden positive Lokalisationen an 3 verschiedenen Tagen und ohne MRSA-wirksame Therapie seit 3 Tagen.

E ... Entlassung aus stationärer Therapie und ambulante Weiterbehandlung.

Welche Aussage ist richtig?

10 Welches ist der häufigste MRSA und anderen nosokomialen Keimen? nosokomialen Infektionen
... bei allen schweren Infektionen durch S. aureus (auch Oxacillin-sensible Stämme).

B ... bei Unverträglichkeit von Clindamycin.

C ... bei Fremdkörper-assoziierten Infektionen mit MRSA.

D ... zur Vermeidung der Resistenzentwicklung gegen Vancomycin.

E ... zur Sequentialtherapie bei leichten Infektionen.

A unsachgemäß aufbereitete Endoskope.

$B$ die Hände des Personals

C Niesen

D Luft

E Gegenstände

A ... Screening von Risikopatienten.

B ... Isolierung von Patienten mit Nachweis von MRSA.

C ... Händedesinfektion des Personals vor und nach Patientenkontakt.

... Therapie von Infektionen und Sanierung des Trägerstatus bei Patienten.

E ... kalkulierte Gabe von Vancomycin bei allen im Krankenhaus erworbenen Infektionen.

\section{Datenschutz}

Ihre Daten werden ausschließlich für die Bearbeitung dieser Fortbildungseinheit verwendet. Es erfolgt keine Speicherung der Ergebnisse über die für die Bearbeitung der Fortbildungseinheit notwendige Zeit hinaus. Die Daten der Evaluation werden an die Nordrheinische Akademie für ärztliche Fort- und Weiterbildung zur wissenschaftlichen Auswertung übermittelt. Die Angaben zur Person dienen nur statistischen Zwecken und werden von den Adressangaben anonymisiert verarbeitet. 